\title{
Printed passive matrix addressed electrochromic displays
}

\author{
Peter Andersson Ersman, Jun Kawahara and Magnus Berggren
}

Linköping University Post Print

N.B.: When citing this work, cite the original article.

Original Publication:

Peter Andersson Ersman, Jun Kawahara and Magnus Berggren, Printed passive matrix addressed electrochromic displays, 2013, Organic electronics, (14), 12, 3371-3378.

http://dx.doi.org/10.1016/j.orgel.2013.10.008

Copyright: Elsevier http://www.elsevier.com/

Postprint available at: Linköping University Electronic Press

http://urn.kb.se/resolve?urn=urn:nbn:se:liu:diva-91270 


\title{
Printed passive matrix addressed electrochromic displays
}

Peter Andersson Ersman ${ }^{a, *}$, Jun Kawahara ${ }^{\text {a,b,c }}$, and Magnus Berggren ${ }^{\text {b }}$

${ }^{\text {a }}$ Printed Electronics, Acreo Swedish ICT AB, Box 787, SE-60117, Norrköping, Sweden

${ }^{\mathrm{b}}$ Organic Electronics, Dept. of Science and Technology, Linköping University, SE-60174, Norrköping, Sweden

${ }^{\mathrm{c}}$ R\&D Strategy Department, Lintec Corporation, 5-14-42 Nishiki-cho, Warabi, Saitama, 3350005, Japan

* Corresponding author: Tel. +46 11202507

E-mail address: peter.andersson.ersman@acreo.se

Keywords: Passive matrix, electrochromic display, printed electronics, PEDOT:PSS

\begin{abstract}
Flexible displays are attracting considerable attention as a visual interface for applications such as in electronic papers and paper electronics. Passive or active matrix addressing of individual pixels require display elements that include proper signal addressability, which is typically provided by non-linear device characteristics or by incorporating transistors into each pixel. Including such additional devices into each pixel element make manufacturing of flexible displays using adequate printing techniques very hard and complicated. Here, we report all-printed passive matrix addressed electrochromic displays (PMAD), built up from a very robust three-layer architecture, which can be manufactured using standard printing tools. Poly(3,4-ethylenedioxythiophene) doped with poly(styrenesulfonate) (PEDOT:PSS) serves as
\end{abstract}


the conducting and electrochromic pixel electrodes and carbon paste is used as the pixel counter electrodes. These electrodes sandwich self-assembled layers of a polyelectrolyte that are confined to desired pixel areas via surface energy patterning. The particular choice of materials results in a desired current vs. voltage threshold that enables addressability in electronic cross-point matrices. The resulting PMAD operates at less than $3 \mathrm{~V}$, exhibits high colour switch contrast without cross-talk and promises for high-volume and low-cost production of flexible displays using reel-to-reel printing tools on paper or plastic foils.

\section{Introduction}

Major efforts are currently being devoted to explore and develop new devices and systems for printed electronics applications [1-7]. The theme of this effort aims at providing novel electronic functionalities on flexible substrates such as plastic foils or papers. Flexible display devices are one of the crucial cornerstones of this research and development effort, and a large variety of different flexible display devices and systems have successfully been demonstrated during the last decade, e.g. electrochromic displays, organic light emitting diode displays and electrophoretic displays [8-12]. Since such devices and display systems most often are manufactured in a complex production flow containing one or several vacuum processing steps, there is still a huge technical barrier to carry out an entire manufacturing process using standard sheet-based or roll-to-roll printing, coating and lamination techniques. Electrochromic (EC) active matrix addressed displays (AMAD) have been explored for various low-end display applications, in particular for areas in which the requirements on e.g. switching time and pixel resolution are a bit relaxed. EC-AMADs, which are built up from pixels including a display element and an addressing transistor, have been demonstrated on either paper or plastic substrates [12-14]. Even though the very same materials have successfully been utilized as the active material in both display elements and transistors, EC- 
AMADs still require a fairly large number of printed layers, thus ruling out simple manufacturing and low cost.

In passive matrix addressed displays (PMAD) addressing transistors are not utilized to provide addressability for the display element. In PMAD configuration, the system complexity is then much lower which enables a relatively much simpler manufacturing scheme. However, one particular drawback of PMADs is that they typically suffer from crosstalk effects, i.e. the applied voltage aiming to update one specific pixel, at the intersection of a certain row and column, also affects and causes coloration of neighbouring non-addressed pixels [15]. There are various ways to circumvent such cross-talk effects in PMADs, for example by combining each pixel with a diode exhibiting rectifying and strong non-linear current vs. voltage (I-V) characteristics [16], but yet again, additional components implies an increased number of processing steps.

Here, we have developed EC pixels exhibiting non-linear coloration vs. addressing voltage characteristics with a threshold voltage at around $\pm 1 \mathrm{~V}$. The EC pixel exhibits good bistability as the addressing voltage is decoupled. The electrode and electrolyte materials were deposited by screen printing and wire bar coating, respectively, on top of flexible plastic substrates and the number of included layers was kept at a minimum; an electrically conducting carbon paste, an ionically conducting polyelectrolyte and an electronically conducting electrochromic polymer served as the counter electrodes, the electrolyte and the pixel electrodes, respectively. This very simple three-layer device architecture was enough to define an entire EC-PMAD containing up to $7 \times 128$ EC pixels. This EC-PMAD, based on the robust and simple device architecture shown in Fig. 1, is promising as the display interface in all-printed electronic systems in applications such as distributed diagnosis, home healthcare or sensor platforms monitoring the status of perishable goods in logistic chains. 


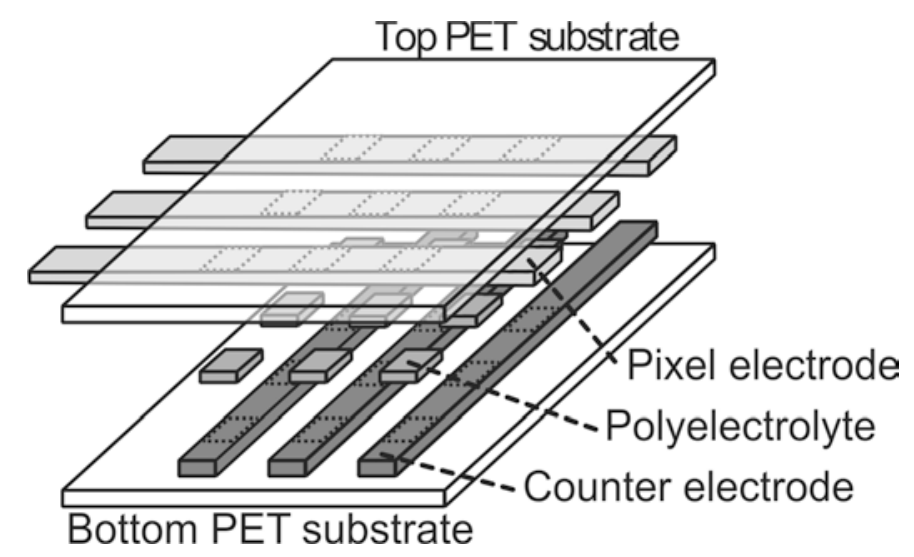

Fig. 1. An illustration showing the simple architecture of a $3 \times 3$ EC-PMAD consisting of 9 EC pixels.

\section{Materials and methods}

\subsection{Materials}

Transparent polyethylene terephthalate (PET, Polifoil Bias) film was purchased from Policrom Screen and was used as the solid substrate carrying the display components. As a top electrode, PET film with pre-coated PEDOT:PSS (Orgacon EL-350) was purchased from AGFA-Gevaert. This product was also used as the counter electrodes for the reference samples. Conducting carbon paste (7102) for screen printing was purchased from DuPont and printed onto the PET substrate by using a 120-34 nylon screen mesh (120 threads/cm, thread diameter $34 \mu \mathrm{m}$ ), followed by drying process at $120^{\circ} \mathrm{C}$ for 5 minutes. An aqueous solution of poly(diallyl dimethyl ammonium chloride) (PDADMAC), purchased from Sigma-Aldrich, was used as the water-based polyelectrolyte. To obtain a white opaque electrolyte, $10 \mathrm{wt} \%$ of $\mathrm{TiO}_{2}$ powder (KRONOS 2300 purchased from KRONOS) was added into the polyelectrolyte solution and mixed carefully before use. The solution of $\mathrm{pH}$ colour indicator (Universal indicator solution 36828, pH 4-10) was purchased from Riedel-de-Haën GmbH. 


\subsection{Electrical characterization of EC pixels}

The PEDOT:PSS layer on the Orgacon film was cut and patterned into an area of $35 \times 35 \mathrm{~mm}^{2}$ by using a knife plotter tool, and the square-shaped pattern was then used as the pixel electrode. A $35 \times 35 \mathrm{~mm}^{2}$ square of conducting carbon was screen printed onto a PET film and used as the counter electrode. At one corner of each electrode, a $5 \times 5 \mathrm{~mm}^{2}$ active area was defined by attaching a spacer consisting of an adhesive plastic film having an area of $20 \times 20$ $\mathrm{mm}^{2}$ with a $5 \times 5 \mathrm{~mm}^{2}$ cavity. The white and opaque electrolyte was deposited manually onto each electrode and dried into a semi-solidified state for 1 minute at $60{ }^{\circ} \mathrm{C}$. After drying, each set consisting of one pixel electrode and one counter electrode was laminated to complete one EC pixel cell.

\subsection{I-V measurement setup in ambient atmosphere}

Each EC pixel device was biased by using an Agilent 4155B Semiconductor Parameter Analyser, and the electric current between the counter and the pixel electrodes was recorded as a function of the applied potential. The voltage was swept in the range of $\pm 2.5 \mathrm{~V}$ at an increment of $10 \mathrm{mV}$ per sample and the time between two samples was set to $20 \mathrm{~ms}$.

\subsection{Measurement setup to determine the switching behaviour of EC pixels}

EC pixels having an area of $1.5 \times 1.5 \mathrm{~mm}^{2}$ were manufactured according to the description above, but they were instead used to observe the colour switching behaviour as a function of the applied potential. The measurement was performed by using a laser diode in conjunction with a photodiode [17]. The display was irradiated by a laser diode peaking at $650 \mathrm{~nm}$, a wavelength that matches the absorption peak of the reduced state of PEDOT:PSS, and the scattered light was detected by a photodiode (S1337-66 from Hamamatsu, 5.8 $\times 5.8 \mathrm{~mm}^{2}$ active area) and the photocurrent was recorded using a Keithley 2400 SourceMeter. 


\subsection{Investigation of the electrochemical capacitor}

Two rectangles $\left(20 \times 5 \mathrm{~mm}^{2}\right)$ of conducting carbon paste 7102 were stencil printed onto a PET substrate. A polyelectrolyte consisting of PDADMAC was mixed with $\mathrm{TiO} 2$ powder and $\mathrm{pH}$ indicator according to a weight ratio of 10/1/0.6 (including solvents). The two carbon rectangles were laterally connected by the polyelectrolyte solution. The I-V characteristics of this carbon/electrolyte/carbon electrochemical capacitor were firstly measured according to the description above. Secondly, a potential difference of $2.5 \mathrm{~V}$ was constantly applied across the carbon electrodes and the colour change of the $\mathrm{pH}$ indicator was observed by microscope.

\subsection{Preparation of PMADs}

A set of conducting carbon counter electrodes consisting of 128 rectangles are deposited in parallel on a PET film by using screen printing technique, where each rectangle has an area of $0.8 \times 30 \mathrm{~mm}^{2}$ with a $0.2 \mathrm{~mm}$ separation gap between two neighbouring rectangles. Separately, a set of PEDOT:PSS-based pixel electrodes was prepared from Orgacon EL-350 film by cutting its surface using a knife plotter, such that the sheet of electrodes consisted of seven rectangles aligned in parallel, where each rectangle has an area of $0.8 \times 140 \mathrm{~mm}^{2}$ and approximately $0.2 \mathrm{~mm}$ separation gap between two rectangles. Along the edge of each 0.8×140 $\mathrm{mm}^{2}$ electrode, a conducting 50-100 $\mu \mathrm{m}$ wide line of silver (U5603 provided by SunChemical) was inkjet printed by using a Dimatix Materials Printer DMP-2800 in order to support the conductivity along the relatively long pixel electrode. The printed layers of conducting carbon and silver were both dried at $120{ }^{\circ} \mathrm{C}$ for 5 minutes. Lines of a polystyrene (PSt) solution (dissolved in toluene, $1 \mathrm{wt} \%$, purchased from Sigma-Aldrich) were then inkjet printed according to a $1 \times 1 \mathrm{~mm}^{2}$ grid-shape pattern along the separation gaps on top of both electrodes. The resulting grid consisted of at least $7 \times 128$ squares, where each line of PSt also covered the inkjet printed silver lines in order to prevent for unwanted electrochemical reactions involving silver. The water-based polyelectrolyte solution was uniformly blade 
coated and then automatically patterned into the cavities between the hydrophobic grid lines; a self-alignment property caused by the difference in surface tension between the electrode material and the PSt lines. The patterned electrolyte layers were semi-solidified at $60{ }^{\circ} \mathrm{C}$ for 1 minute, and each PMAD was finally completed by laminating the two electrode substrates such that $7 \times 128$ EC pixels were created. Surface energy patterning is a preferred method since the requirement on accurate registration between subsequently printed layers is cancelled, and lamination is an advantageous technique by that it automatically brings a protective encapsulation layer. A schematic of a PMAD containing 3×3 EC pixels is shown in Fig. 1 .

\subsection{Driving of PMADs}

Various kinds of electronic circuits have successfully been tested to verify the addressability of unique EC pixels in the PMAD. Examples of digital solutions are the open-source electronic platform provided by Arduino, or the combination of a demultiplexer and D-type flip-flops (74HC4067 and 74HC174 from NXP Semiconductors) connected to the rows and columns, respectively. A solution based on analogue signals was also verified by using a data acquisition card with 32 analogue outputs (DAQ PCI-6723 controlled by LabVIEW software, both from National Instruments) connected to the rows and columns of the PMAD.

\section{Results and discussion}

\subsection{I-V characteristics of EC pixel devices in ambient atmosphere}

Carbon-based counter electrodes have previously been used in various applications related to iconic electrochromic displays and smart windows $[18,19]$. Carbon is considered to be an advantageous counter electrode material in electrochemical devices for several other reasons; its durability and stability has been proven excellent in commercial electrolyte capacitors, its resulting electric double layer have high charge storage capacity independently of the polarity 
of the charges, and counter electrodes consisting of carbon can be deposited using several common patterning methods, such as screen printing. Additionally, the possibility to obtain low-cost manufacturing is enabled by the combination of low-cost deposition methods and the fact that carbon paste is much more inexpensive as compared to most other conducting materials. In the present work, the carbon electrode, when combined with suitable polyelectrolyte and EC material, provide also a desired threshold voltage that provides unique pixel addressability.

Fig. 2 shows the electric current vs. the applied voltage across two kinds of individual EC pixel configurations; one EC pixel created according to the three-layer architecture shown in Fig. 1, and one reference EC pixel. Fig. 2(a) shows the reference EC pixel where both the counter and the pixel electrodes are composed of poly(3,4-ethylenedioxythiophene) (PEDOT) doped with poly(styrene sulfonate) (PSS), PEDOT:PSS [20], which results in a typical capacitive charging behaviour. Hence, the electrochemical redox reaction as well as the pixel coloration occurs linearly throughout the whole addressing potential window, i.e. the charging current is constant for different $\mathrm{V}_{\mathrm{P}}$. Such linear coloration disables utilization of this EC pixel in PMADs, simply due to the fact that a threshold voltage $\left(\mathrm{V}_{\mathrm{TH}}\right)$ is lacking to prevent crosstalk coloration of neighbouring pixels within the PMAD. PEDOT:PSS is not only an electrochromic polymer, but it also changes its electrical conductivity upon altering its oxidation state, which therefore explains the rapid decline of the current level on the positive side of each voltage sweep, i.e. when the PEDOT:PSS serving as the pixel electrode becomes dark blue and semi-conducting. In contrast, when the device has a conducting carbon layer as its counter electrode, a rather different behaviour of the I-V characteristic of the EC pixel is shown. During the first half of the potential sweep, i.e. when the pixel switches from its white-coloured state to the blue-coloured state, a fairly small current can be observed until the applied potential reaches $\mathrm{V}_{\mathrm{TH}^{+}} \sim 1.0 \mathrm{~V}$, which then results in a rapid increase of the current level, see Fig. 2(b). The current ratio for $\mathrm{V}_{\mathrm{P}}=0 \mathrm{~V}$ and $\mathrm{V}_{\mathrm{P}}=2.0 \mathrm{~V}$ is almost $100(\sim 0.5 \mu \mathrm{A}$ vs. 
$\sim 50 \mu \mathrm{A})$. Such strong non-linear effect implies that the blue-coloration of the EC pixel does not occur to any large extent below the $\mathrm{V}_{\mathrm{TH}^{+}}$of $1.0 \mathrm{~V}$. Similarly, the second half of the updating cycle shows a negative $\mathrm{V}_{\mathrm{TH}}$ of at least $-1.0 \mathrm{~V}\left(\mathrm{~V}_{\mathrm{TH}}\right)$, hence, the bi-stability of the EC pixel is improved by that the blue-coloured state remains also at $\mathrm{V}_{\mathrm{P}}=0 \mathrm{~V}$. The capability of colour retention, for both polarities of the applied $V_{\mathrm{P}}$, is a fundamental requirement in order to enable the scheme of passive matrix addressing. Observations on how the colour contrast varies as a function of the applied EC pixel voltage will be discussed later. Different counter electrode materials, such as gold, silver, polyaniline and other carbon pastes, as well as various electrolytes, e.g. polyelectrolytes, ionic liquids and polymer electrolytes containing various kinds of salts, have been evaluated in combination with a PEDOT:PSS-based pixel electrode, and it is only the combination of a carbon-based counter electrode and a polyelectrolyte that results in the desired threshold voltage and thereby enabling addressability in PMADs.
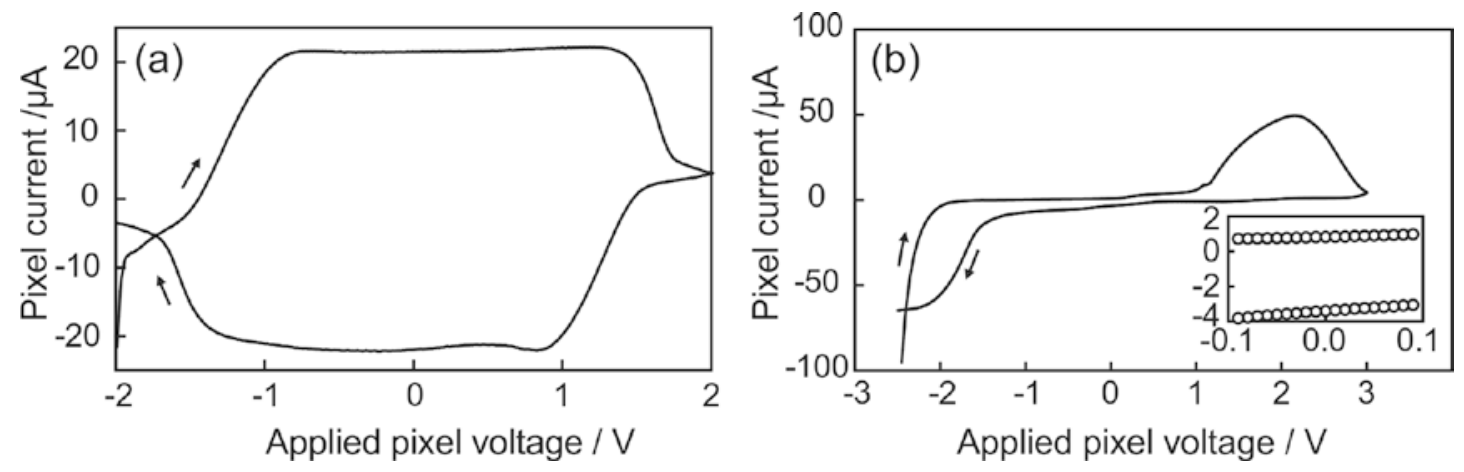

Fig. 2. The I-V characteristics of different EC pixel architectures. (a) The I-V characteristics of a reference EC pixel, in which no threshold voltage is observed and hence no unique addressability can be obtained, when using PEDOT:PSS as both pixel and counter electrodes. (b) The I-V characteristics of an EC pixel, in which the threshold voltage is observed such that unique pixel addressability can be obtained, when using PEDOT:PSS and carbon paste as the pixel and counter electrode, respectively. The inset shows the current levels at an applied voltage of around $0 \mathrm{~V}$. 


\subsection{Optical investigation of the non-linear EC pixel coloration}

The optical coloration behaviour of the two EC pixels is given in Fig. 3(a), where the counter electrode consists of PEDOT:PSS and carbon, respectively. Here the signal detected by the photodiode and the voltage applied across the EC pixels are plotted as a function of time. The photocurrent is varied and depends on the number of reflected photons from the active pixel surface. The pixel surface was irradiated with a laser diode emitting at $650 \mathrm{~nm}$. A low photocurrent is then recorded if the EC pixel is switched to its deep blue-coloured state, and vice versa. The colour switching characteristics, i.e. the photocurrent, of the EC pixel is almost perfectly linear when both electrodes are composed of PEDOT:PSS. On the other hand a strong non-linear colour switch characteristic is obtained when the counter electrode consists of conducting carbon. A clear coloration threshold voltage of $\mathrm{V}_{\mathrm{P}} \sim 1.0 \mathrm{~V}$ is found and the colour switching saturates at $\mathrm{V}_{\mathrm{P}} \sim 2.0 \mathrm{~V}$; a behaviour that is in good agreement with the non-linear I-V characteristics given in Fig. 2(b).

The time it takes to switch the EC pixel from the decoloured state to the coloured state was monitored upon applying a sufficiently high voltage; an amplitude that is high enough to cause a full reduction of the PEDOT:PSS pixel electrode layer directly from its oxidised state, i.e. $-2 \mathrm{~V}$ to $+2 \mathrm{~V}$ without any gradual steps. Fig. 3(b) shows that the colour change of the EC pixel saturates within roughly $1 \mathrm{~s}$. This switching time is slightly longer than what is recorded for a symmetric EC pixel consisting of PEDOT:PSS in both electrodes [17], but still feasible for utilization in PMADs. The applicability of using the EC pixel in PMADs has been further verified by extensive cycling tests, in which the desired threshold voltage and colour switch characteristics remain almost constant for several thousand cycles, see Figure 3(c). 

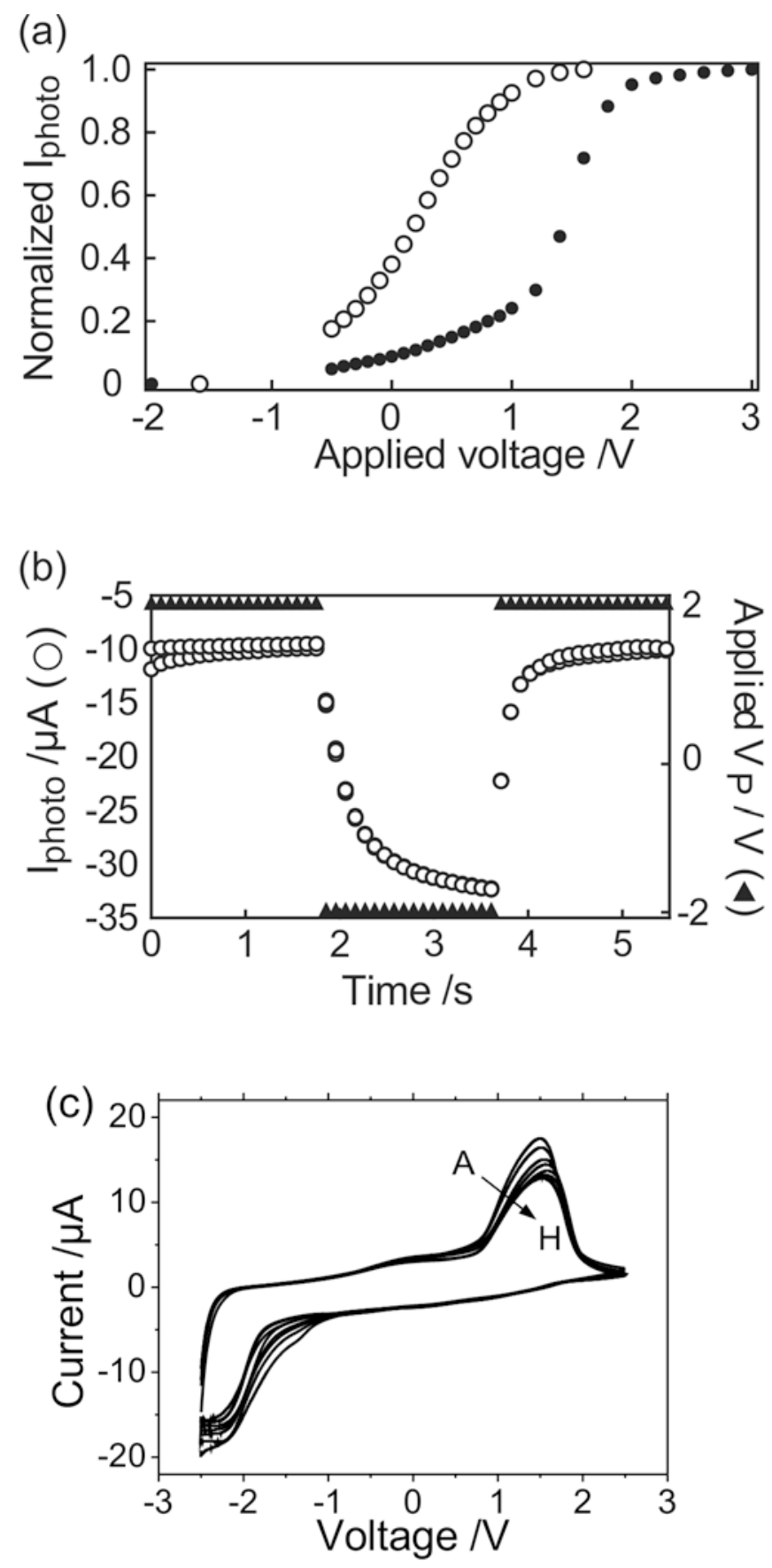

Fig. 3. Optical investigation revealing the coloration behaviour and switching time of the EC pixels. (a) The coloration behaviour in EC pixels having the counter electrodes composed by PEDOT:PSS ( $\circ$ ) and conducting carbon $(\bullet)$, respectively. The $\mathrm{V}_{\mathrm{P}}$ sweep started by applying a negative voltage to the counter electrode, which results in oxidised pixel electrodes. $V_{P}$ was then swept $\left(0.1 \mathrm{~V} / \mathrm{step}\right.$ until $\mathrm{V}_{\mathrm{P}}=1.0 \mathrm{~V}$ and $0.2 \mathrm{~V} / \mathrm{step}$ at $\left.\mathrm{V}_{\mathrm{P}}>1.0 \mathrm{~V}\right)$ towards positive voltages and thereby gradually reducing the pixel electrodes. The time between two samples 
was $1 \mathrm{~s}$. (b) The switching time is measured in a $2 \times 2 \mathrm{~mm}^{2}$ EC pixel with a carbon counter electrode. Open circles $(\circ)$ and filled triangles $(\boldsymbol{\Delta})$ correspond to photocurrent and $\mathrm{V}_{\mathrm{P}}$, respectively. The negative voltage in the graph corresponds to oxidation of the pixel electrode to its white coloured state, while the pixel is reduced to its blue coloured state by the opposite voltage polarity. The time between two samples was 0.1 s. (c) The EC pixel switching characteristics remain almost constant upon continuous cycling; [A-H] corresponds to the behaviour after [111, 188, 272, 352, 724, 1009, 2689, 3724] cycles, respectively.

\subsection{Investigation of the interfaces between the electrodes and the electrolyte}

The $\mathrm{pH}$ colour indicator used in this report changes its colour from red at low $\mathrm{pH}$ to violet for high $\mathrm{pH}$, via yellow, green (neutral $\mathrm{pH}$ ) and blue. Microscope images were recorded upon applying certain voltages across an electrochemical cell, or capacitor, that consists of two carbon electrodes bridged by a polyelectrolyte, see Fig. 4. After 3 minutes the electrolyte layer on top of the positively biased electrode showed red colour, while the negatively biased side appeared blue. One plausible explanation for this result is that protons $\left(\mathrm{H}^{+}\right)$, or hydronium cations $\left(\mathrm{H}_{3} \mathrm{O}^{+}\right)$, and hydroxide anions $\left(\mathrm{OH}^{-}\right)$are generated at the carbon electrodepolyelectrolyte interfaces, respectively. This result indicates that the threshold voltage observed in the EC pixel could originate from the following set of electrochemical halfreactions, which only occur when $\mathrm{V}_{\mathrm{P}}$ exceeds $\mathrm{V}_{\mathrm{TH}+}$ :

Cathode: PEDOT $^{+}$PSS $^{-}+\mathrm{M}^{+}+\mathrm{e}^{-} \rightarrow$ PEDOT $^{0}+$ PSS $^{-}{ }^{+}$

Anode: $2 \mathrm{H}_{2} \mathrm{O} \rightarrow 4 \mathrm{H}^{+}+\mathrm{O}_{2}+4 \mathrm{e}^{-}$

The protons are generated to an amount so that the colour of the $\mathrm{pH}$ indicator is changed from green to red, i.e. the $\mathrm{pH}$ is decreased in the vicinity of the counter electrode. This result is also accompanied with that the EC pixel becomes blue-coloured. Conversely, when $\mathrm{V}_{\mathrm{P}}$ is more 
negative than $\mathrm{V}_{\mathrm{TH}}$-, the PEDOT reaction stated above is reversed and the oxidised transparent state is reached together with that $\mathrm{OH}^{-}$anions are generated at the counter electrode:

Cathode: $2 \mathrm{H}_{2} \mathrm{O}+2 \mathrm{e}^{-} \rightarrow \mathrm{H}_{2}+2 \mathrm{OH}^{-}$

This increase of the surrounding $\mathrm{pH}$ at the counter electrode is monitored by a colour shift to green-blue of the $\mathrm{pH}$ indicator.

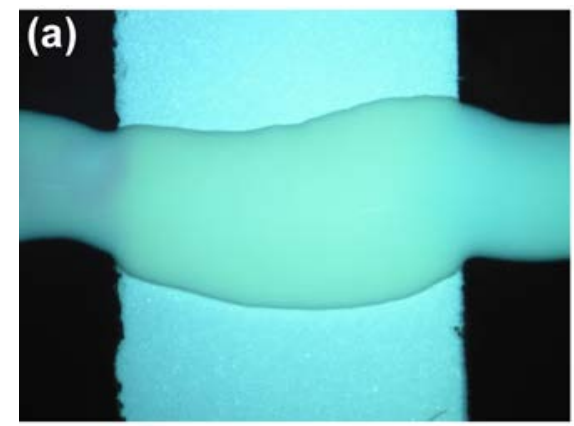

(b)

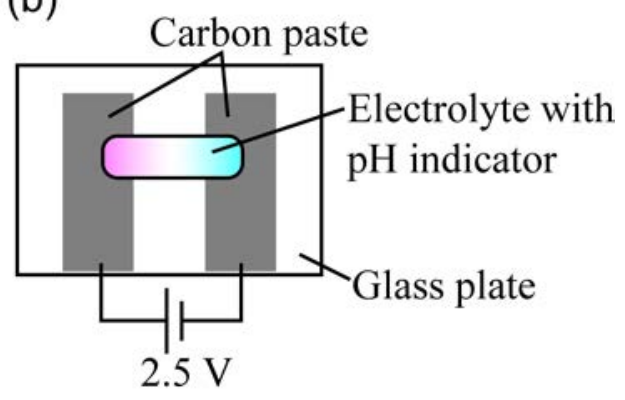

Fig. 4. (Colour online). Confirmation of electrolysis inside a lateral electrochemical capacitor structure. (a) A microscope image showing the colour change of the $\mathrm{pH}$ indicator in an electrochemical capacitor consisting of two carbon electrodes ionically connected by a polyelectrolyte. (b) The electrochemical capacitor is drawn schematically. The overlap area between the electrolyte and each carbon electrode is approximately $5 \times 5 \mathrm{~mm}^{2}$.

The current through the lateral electrochemical capacitor shown in Fig. 4(b) was recorded as a function of time while two different voltages were applied, below (0.5 V) and above (2.5 V) the $\mathrm{V}_{\mathrm{TH}+}$, respectively. Also, for comparison, a vertical electrochemical capacitor based on 
two $5 \times 5 \mathrm{~mm}^{2}$ carbon electrodes sandwiching an electrolyte was concurrently prepared. Both the vertical and lateral electrochemical capacitor devices showed similar current vs. time characteristics; a steady-state current level was reached subsequently to an initial ( 2 s) capacitive charging peak, see Table 1 . For the $0.5 \mathrm{~V}$ case, i.e. below $\mathrm{V}_{\mathrm{TH}+}$, the steady-state current and the accumulated charge, recorded during $300 \mathrm{~s}$, were both relatively low, while they were increased by at least 800 and 300 times for the lateral and vertical configuration, respectively, upon increasing the voltage to $2.5 \mathrm{~V}$, i.e. above the $\mathrm{V}_{\mathrm{TH}+}$. Such strong non-linear increase in the $\mathrm{I}-\mathrm{V}$ characteristics in conjunction with the $\mathrm{pH}$ change given in Fig. 4 is a strong indication of electrolysis. Further, the same type of current vs. time and voltage measurement was carried out on yet another vertical device having the same active electrode area $\left(5 \times 5 \mathrm{~mm}^{2}\right)$, but in which one of the carbon electrodes was replaced by a PEDOT:PSSbased pixel electrode to form an EC pixel. Overall, this device exhibits similar device characteristics as compared to the two symmetric carbon-based electrochemical capacitor architectures. However, for an applied voltage below the $\mathrm{V}_{\mathrm{TH}+}$, an elevated amount of charge $(15.5 \mu \mathrm{C})$ is recorded. This elevated charging may be explained by ion exchange between the electrolyte and the PEDOT:PSS electrode. However, the associated colour change due to this parasitic charging of the EC pixel is not discernible to the eye. For all the three devices reported in Table 1, regardless of device architecture, an applied voltage below $\mathrm{V}_{\mathrm{TH}}$ generates a relatively low steady-state current level. This is in agreement with a very slow reaction rate of water splitting. Further, below $\mathrm{V}_{\mathrm{TH}}$ the amount of charge that was delivered during $300 \mathrm{~s}$ is insufficient to cause any major EC pixel coloration. On the contrary, above the threshold the rate of electrolysis is high enough, as evidenced by an increase of both steady-state current and the accumulated charge, which enables full EC pixel coloration. So, the results here presented suggests that electrolysis is responsible for the strong non-linear charging and coloration characteristics of the EC pixel device built up from PEDOT:PSS and carbon electrodes. 
Table 1. Steady-state current and charge measured in the electrochemical capacitors. ${ }^{1)}$ All active areas are $5 \times 5 \mathrm{~mm}^{2} .{ }^{2)}$ See Fig. 4 (b) for the device architecture. ${ }^{3)}$ Current recorded 300 seconds after starting the measurement. ${ }^{4)}$ Time integration of the current between 0 and $2 \mathrm{~s}$. Note that the steady-state current levels of the vertical capacitor is lower as compared to the lateral ditto, which is explained by a slower reaction rate due to less water content in this particular electrolyte. Additionally, the relatively low current in the EC pixel at $2.5 \mathrm{~V}$ is explained by the lowered conductivity in the reduced state of PEDOT:PSS.

\begin{tabular}{|l|c|c|c|c|}
\hline \multirow{2}{*}{ Device ${ }^{1)}$} & \multicolumn{2}{|c|}{ Steady-state current $(\mu \mathrm{A})^{3)}$} & \multicolumn{2}{c|}{ Charge $(\mu \mathrm{C})^{4)}$} \\
\cline { 2 - 5 } & $0.5 \mathrm{~V}$ & $2.5 \mathrm{~V}$ & $0.5 \mathrm{~V}$ & $2.5 \mathrm{~V}$ \\
\hline Capacitor (lateral) $^{2)}$ & 0.027 & 22 & 0.39 & 109 \\
\hline Capacitor (vertical) & $1 \times 10^{-4}$ & 8.4 & 0.41 & 132 \\
\hline EC pixel (vertical) & 0.014 & 1.1 & 15.5 & 205 \\
\hline
\end{tabular}

In an EC pixel, the pixel electrode switching is considered to be a semi-reversible electrochromic reaction, as indicated by that the colour switching activity diminishes after a few thousand switch cycles. This effect is however considered as a temporary exhaustion, caused by the continuous cycling, rather than true degradation. Hence, the colour switching behaviour with maintained threshold voltage is regained by delaminating the EC pixel, adding a small drop of deionized water and finally re-laminating the two sheets again. In other words, as fresh electrolyte is introduced into the device, the proper device operation is retrieved. Here, it should be noted that many printed electronic applications only require a relatively low number of switch cycles in terms of operational lifetime, i.e. less than a few thousand switch cycles, and/or a non-continuous updating sequence, where the latter would result in enhanced device lifetime caused by the hygroscopic property of the polyelectrolyte. 


\subsection{Driving PMADs}

Displays consisting of EC pixels, which exhibit a threshold voltage feature, can be operated by various passive matrix-addressing protocols. One alternative that successfully has been implemented is to use a programmable circuit with a number of digital outputs, where each output is connected to a row or column on the display device, respectively. The advantage of using digital circuitry is that a high impedance state can be easily implemented on nonaddressed rows or columns, such that the display can be updated without any cross-talk effects. A similar approach is obtained by using the same programmable circuit for the columns and another digital circuit capable of demultiplexing the row lines. Other addressing protocols rely on analogue signals, where the threshold voltage of each EC pixel still ensures that an image can be presented without cross-talk effects, for example by using the V/3 or the V/2 addressing protocols [21]. The schematic model of the V/3 addressing protocol is illustrated in Fig. 5. Here, the voltage required for switching an EC pixel to its blue colour state is represented by the letter "V". The PEDOT:PSS rows were exposed to a signal, one by one, with a pulse width of e.g. 1 second, that is, all desired columns of the corresponding row were biased at the same time. The colour switch potential was set to $3 \mathrm{~V}$, and unique EC pixel addressing is obtained by applying $0 \mathrm{~V}$ to the addressed row, $2 \mathrm{~V}$ to non-addressed rows, $1 \mathrm{~V}$ to non-addressed columns and $3 \mathrm{~V}$ to addressed columns. The V/3-protocol is advantageous in the sense that a lower voltage is required in order to obtain unique addressability, i.e. the threshold voltage must be at least one third of the updating voltage $\mathrm{V}$, and by that only one voltage polarity is used. The disadvantage of this protocol is that 4 different amplitudes are required; $0, \mathrm{~V} / 3,2 \mathrm{~V} / 3$ and $\mathrm{V}$. The V/2-protocol, on the other hand, is advantageous by that only 3 voltage amplitudes are used, $0,-\mathrm{V} / 2$ and $\mathrm{V} / 2$, while it is disadvantageous by that the requirement on the threshold voltage is higher, and by that both positive and negative addressing voltages are required from the external addressing electronics. The PMADs reported herein have successfully been updated by digital addressing circuits, with and 
without row demultiplexing and by using both the $V / 2$ and the $V / 3$ analogue addressing protocols. It has also been verified that PMADs consisting of PEDOT:PSS-based pixel and counter electrodes, i.e. devices that are lacking the necessary threshold voltage feature, cannot be updated without severe cross-talk effects, and this is independent on the addressing protocol chosen.

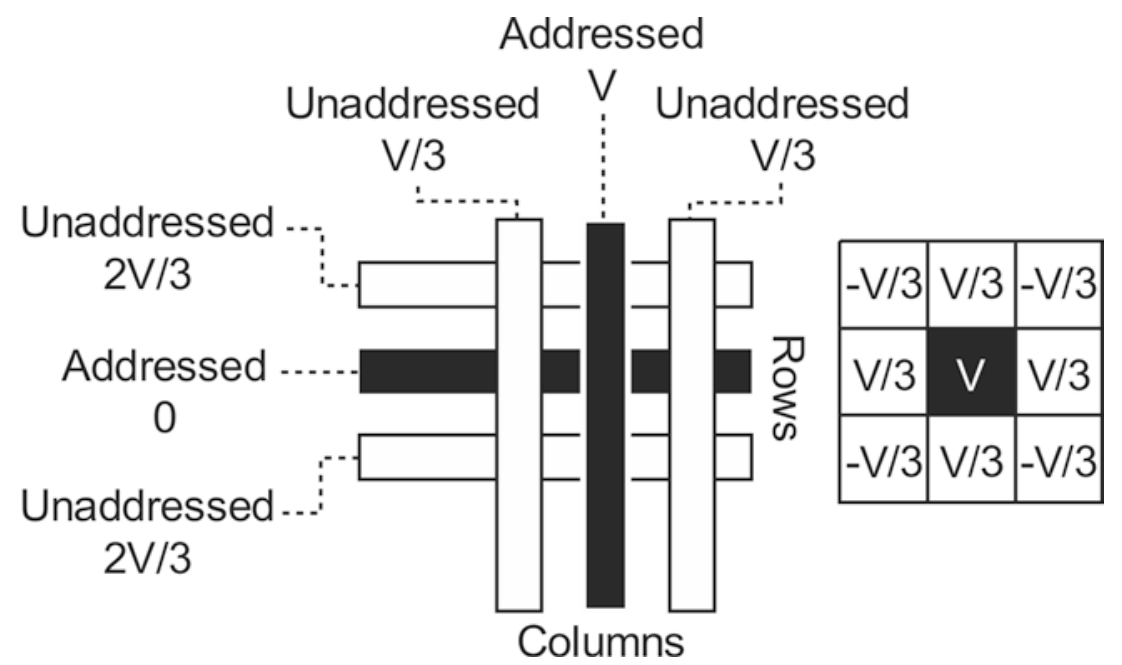

Fig. 5. The V/3 addressing protocol is illustrated. A schematic of the V/3 addressing protocol in a $3 \times 3$ PMAD is shown as well as the resulting image in the $3 \times 3$ PMAD when only the pixel at the centre of the display is activated by applying $+\mathrm{V}$, while the rest of the pixels are sensing either $+\mathrm{V} / 3$ or $-\mathrm{V} / 3$, which is below the EC pixel threshold voltage. The $\mathrm{V} / 2$ addressing protocol (schematic not shown) is using $\mathrm{V} / 2$ on the addressed column, $-\mathrm{V} / 2$ on the addressed row and $0 \mathrm{~V}$ on all other rows and columns.

The number of involved materials, along with the number of printed layers, must be kept at a minimum in order to obtain the most straightforward manufacturing route of electronic functionalities on flexible substrates. This has proven to be more challenging than expected since many printed electronic devices reported in the literature yet are relying on complex 
multilayer architectures, which in turn typically implies the requirement on high accuracy registration. However, the simplicity of the passive matrix addressed display reported herein is of crucial importance; only three different materials as well as three printed layers are required, which results in a simplified manufacturing process. Fig. 6(a-d) shows a PMAD demonstrator containing 7 rows and 128 columns such that alphanumeric characters can be presented. All 896 EC pixels in the resulting PMAD could be uniquely updated without any perceptible cross-talk effects. It should be noted that 50-100 $\mu$ m wide silver lines were inkjet printed in this device, not to obtain the functionality but to shorten the switching time by minimizing the potential drop along the PEDOT:PSS-based conducting rows. A video clip showing fast updating without cross-talk of a PMAD containing $16 \times 16$ EC pixels can be found in the online version of this article; a display device that is illustrated by the static image in Fig. 6(e).

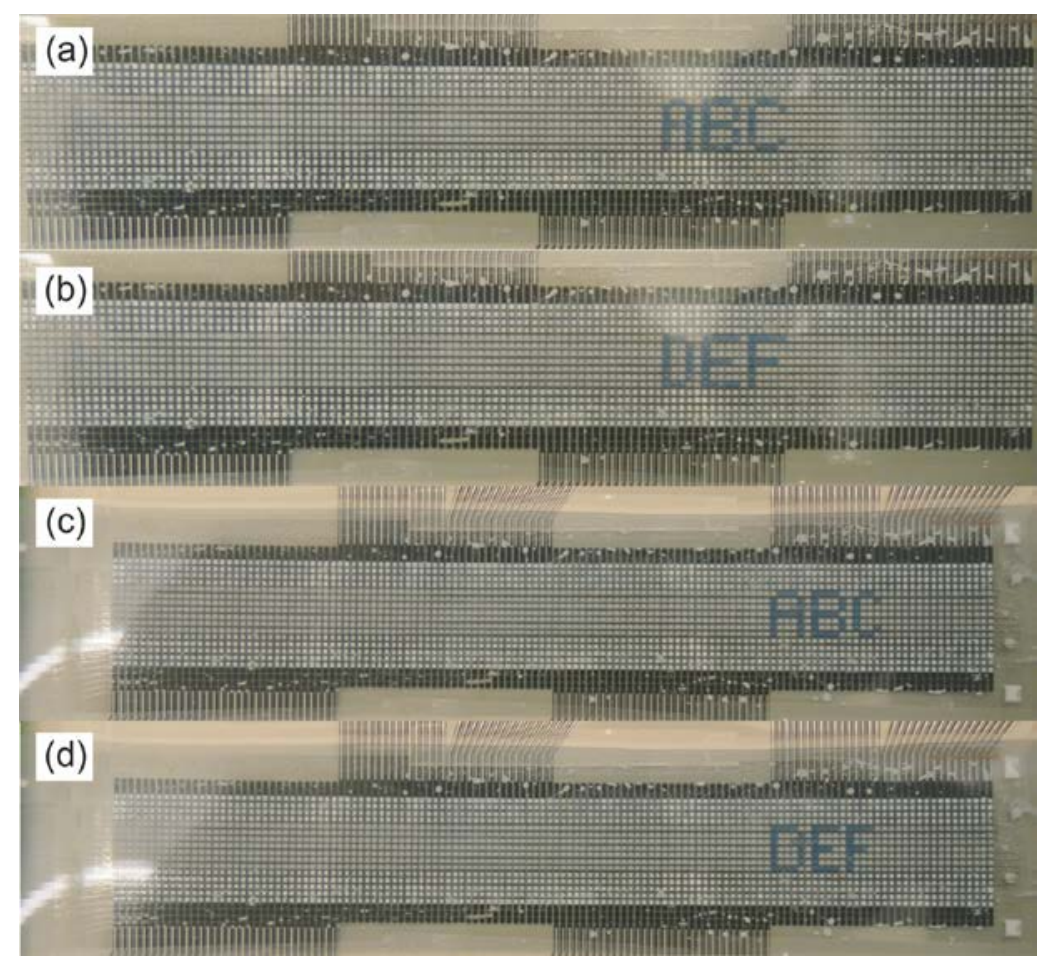




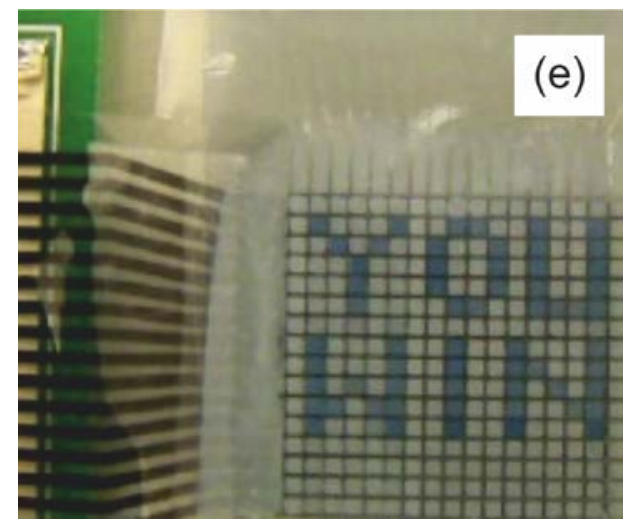

Fig. 6. (Colour online). Photographs showing the successful operation of a $7 \times 128$ PMAD. This particular display was updated by using the V/3 addressing protocol, where $3 \mathrm{~V}$ and $-2 \mathrm{~V}$ was applied for the coloration and the decoloration sequence, respectively. The images in (a) and (b) prove that an arbitrary message can be presented on the display, "ABC” vs. "DEF", while the same messages are shown in another location of the PMAD in (c) and (d). The remaining areas were subsequently tested to verify unique addressability of each EC pixel within the PMAD. Each pixel has an area of $0.9 \times 0.9 \mathrm{~mm}^{2}$ and the pixel pitch is $1 \mathrm{~mm}$, i.e. the fill factor of the resulting PMAD is $81 \%$. (e) The video clip in the online version of this article shows the updating sequence of a printed passive matrix addressed display containing $16 \times 16$ electrochromic pixels. The updating was performed without cross-talk effects by using the analogue signals from an external addressing circuit (DAQ PCI-6723 controlled by LabVIEW) according to the V/2 addressing protocol. The scan rate was $100 \mathrm{~ms}$ per row, the updating voltage was $2.4 \mathrm{~V}$, i.e. $\pm 1.2 \mathrm{~V}$, and the message "YOU WIN" could be observed after a few cycles.

\section{Conclusions}

Passive matrix addressed electrochromic displays manufactured by printing techniques is demonstrated. The addressability of unique electrochromic pixels in the resulting display is obtained by the introduction of a threshold voltage that is generated by combining a carbon- 
based counter electrode with a PEDOT:PSS-based pixel electrode that together sandwiches a polyelectrolyte. The threshold voltage that enables control of pixel charging, and thus also coloration, is governed by electrolysis. Simple manufacturing is ensured by that only three different materials are required in order to obtain the desired device functionality, and a novel method to achieve self-alignment of the electrolyte layer further relaxes the demand for tough registration between the different layers. Hence, the number of materials and processing steps are kept at a minimum, which in turn offer the possibility to achieve low-cost manufacturing of matrix-addressed displays at high production yields. Further development work of the electrolyte is currently being performed in order to improve printability and curability, which in turn is aiming towards all-printed passive-matrix displays manufactured by roll-to-roll techniques.

\section{Acknowledgements}

Prime funding for this project was provided by Lintec Corporation. Also, the authors wish to thank the Centerprise project at VINNOVA (research and innovation for sustainable growth in Sweden). 


\section{References}

[1] Y. Liu, T. T. Larsen-Olsen, X. Zhao, B. Andreasen, R. R. Søndergaard, M. Helgesen, K. Norrman, M. Jørgensen, F. C. Krebs, X. Zhan, Sol. Energ. Mat. Sol. C. 112 (2013) 157-162.

[2] H.-Y. Tseng, V. Subramanian, Org. Electron. 12 (2011) 249-256.

[3] S. K. Park, Y. H. Kim, J. I. Han, J. Phys. D Appl. Phys. 42 (2009) 125102.

[4] R. R. Søndergaard, M. Hösel, M. Jørgensen, F. C. Krebs, J. Polym. Sci. Pol. Phys. 51 (2013) 132-136.

[5] P. Chen, Y. Fu, R. Aminirad, C. Wang, J. Zhang, K. Wang, K. Galatsis, C. Zhou, Nano Lett. 11 (2011) 5301-5308.

[6] A. C. Hübler, M. Bellmann, G. C. Schmidt, S. Zimmermann, A. Gerlach, C. Haentjes, Org. Electron. 13 (2012) 2290-2295.

[7] U. Zschieschang, M. J. Kang, K. Takimiya, T. Sekitani, T. Someya, T. W. Canzler, A. Werner, J. Blochwitz-Nimoth, H. Klauk, J. Mater. Chem. 22 (2012) 4273-4277.

[8] Q. Niu, Y. Shao, W. Xu, L. Wang, S. Han, N. Liu, J. Peng, Y. Cao, J. Wang, Org. Electron. 9 (2008) 95-100.

[9] G. H. Gelinck, H. E. A. Huitema, E. van Veenendaal, E. Cantatore, L. Schrijnemakers, J. B. P. H. van der Putten, T. C. T. Geuns, M. Beenhakkers, J. B. Giesbers, B.-H. Huisman, E. J. Meijer, E. M. Benito, F. J. Touwslager, A. W. Marsman, B. J. E. van Rens, D. M. de Leeuw, Nat. Mater. 3 (2004) 106-110.

[10] Y. Watanabe, K. Nakamura, N. Kobayashi, Phys. Chem. Chem. Phys. 13 (2011) 1942019426.

[11] A. A. Argun, M. Berard, P.-H. Aubert, J. R. Reynolds, Adv. Mater. 17 (2005) 422-426.

[12] P. Andersson, D. Nilsson, P.-O. Svensson, M. Chen, A. Malmström, T. Remonen, T. Kugler, M. Berggren, Adv. Mater. 14 (2002) 1460-1464.

[13] P. Andersson, R. Forchheimer, P. Tehrani, M. Berggren, Adv. Funct. Mater. 17 (2007) 30743082. 
[14] J. Kawahara, P. Andersson Ersman, D. Nilsson, K. Katoh, Y. Nakata, M. Sandberg, M. Nilsson, G. Gustafsson, M. Berggren, J. Polym. Sci. Pol. Phys. 51 (2013) 265-271.

[15] A. E. Aliev, H. W. Shin, Displays 23 (2002) 239-247.

[16] C. Brabec, J. Hausch, Electrochromic Passive Matrix Display Device, Patent publication number WO/2006/051056, Germany, 2006.

[17] J. Kawahara, P. Andersson Ersman, I. Engquist, M. Berggren, Org. Electron. 13 (2012) 469474.

[18] M. O. M. Edwards, G. Boschloo, T. Gruszecki, H. Pettersson, R. Sohlberg, A. Hagfeldt, Electrochim. Acta 46 (2001) 2187-2193.

[19] T. Kubo, J. Tanimoto, M. Minami, T. Toya, Y. Nishikitani, H. Watanabe, Solid State Ionics 165 (2003) 97-104.

[20] L. Groenendaal, F. Jonas, D. Freitag, H. Pielartzik, J. R. Reynolds, Adv. Mater. 12 (2000) 481-494.

[21] F. L. E. Jakobsson, Charge Transport Modulation in Organic Electronic Diodes, Ph.D. thesis, Linköping University, LiU-Tryck, Linköping, Sweden, 2008. 